\title{
Management of the dry eye in Sjogren's syndrome
}

\author{
JOHN WILLIAMSON, W. MALGOLM DOIG, J. V. FORRESTER, \\ M. H. THAM, T. WILSON, KEITH WHALEY, AND W. CARSON DIGK \\ Eye Department, Southern General Hospital, Glasgow $G_{51}{ }_{4} T F$
}

In this paper we report the efficacy of carefully controlled standard forms of treatment in the keratoconjunctivitis (KCS) of Sjøgren's syndrome (SS). Since it is accepted that $\mathrm{SS}$ is a chronic benign inflammatory disease characterized by remissions and exacerbations, the results of this study have been withheld until several years have elapsed. For this reason also we make no attempt to comment on forms of treatment such as hydrophilic contact lenses (Gasset and Kaufman, I97 I Krejci, 1972) or motorized fluid delivery from reservoir glasses (Dohlman, 197I) which as yet we have not utilized in a large series of patients.

Since there is at present nothing that will eradicate KCS in Sjøgren's syndrome, our aim has been to produce relief of symptoms, reduction in signs, and thus a reduced incidence of irreversible ocular damage over a long period of time.

\section{Patients studied}

$9^{8}$ patients suffering from KCS were supervised for no less than 5 years. All were suffering from definite rheumatoid arthritis according to the American Rheumatism Association criteria (Ropes, Bennett, Cobb, Jacox, and Jessar, 1959). There were 88 females and ten males. The mean age was $58 \cdot 4$ yrs (range 32 to 74 ).

Seventy of the patients were cases diagnosed for the first time by one of us (J.W.); the remaining 28 were referred by ophthalmic colleagues from various parts of the West of Scotland. A diagnosis of KCS was made when a forced Schirmer tear test using standardized tear strips (Halberg and Berens, Contactisol, Lindenhurst, U.S.A.) with 10 per cent. ammonia inhalation for 5 min. showed less than $15 \mathrm{~mm}$. of wetting, and I per cent. rose Bengal dye caused at least minimal staining of the interpalpebral conjunctivae (Williamson, Cant, Mason, Greig, and Boyle, 1967).

\section{Methods of assessment}

Progress was determined by change in symptoms and slit-lamp signs, using Tables I and II as guides. The factors shown in these Tables were compiled in a parallel study on the diagnosis of KCS which has been published elsewhere (Williamson, Whaley, Dick, and Anderson, r97ra).

\section{Therapy}

Four treatment regimens were used:
(a) Replacement therapy;
(b) Conservation of tears;
(c) Mucolytic agents;
(d) Hygiene, antibiotics, and topical steroids. 
Table I Incidence (per cent.) of ten symptoms in keratoconjunctivitis sicca

\begin{tabular}{|c|c|}
\hline Symptoms & $\begin{array}{l}\text { Percentage } \\
\text { present }\end{array}$ \\
\hline I. Foreign body sensation & $88 \cdot 0$ \\
\hline 2. Burning & $75 \cdot 0$ \\
\hline $\begin{array}{l}\text { 3. Tiredness, with or without difficulty in opening } \\
\text { the eyes }\end{array}$ & $70 \cdot 0$ \\
\hline $\begin{array}{l}\text { 4. Dry feeling, with or without a poor response to } \\
\text { physical or chemical irritants and emotion }\end{array}$ & $65 \cdot 0$ \\
\hline 5. Redness & $47 \cdot 0$ \\
\hline 6. Difficulty in seeing & $40 \cdot 0$ \\
\hline 7. Itch & $37 \cdot 5$ \\
\hline 8. Aching, soreness, or pain & $37 \cdot 5$ \\
\hline 9. Photosensitivity & $25 \cdot 0$ \\
\hline Io. Excess of secretion, watery, ropy, or film & $22 \cdot 5$ \\
\hline
\end{tabular}

Table II Biomicroscopy changes in keratoconjunctivitis sicca (40 patients)

\begin{tabular}{|c|c|c|c|c|}
\hline \multicolumn{2}{|l|}{ Stain } & \multirow{4}{*}{$\begin{array}{l}\text { Site } \\
\overline{\text { Conjunctiva }}\end{array}$} & \multirow{4}{*}{$\begin{array}{l}\text { Change observed } \\
\text { Dilated interpalpebral vessels } \\
\text { Mild pericorneal injection } \\
\text { Mucus in conjunctival sac }\end{array}$} & \multirow{2}{*}{$\begin{array}{l}\begin{array}{l}\text { Percentage } \\
\text { showing change }\end{array} \\
77.5\end{array}$} \\
\hline \multirow[t]{16}{*}{ Rose Bengal } & \multirow[t]{7}{*}{ Before } & & & \\
\hline & & & & $52 \cdot 5$ \\
\hline & & & & $25 \cdot 0$ \\
\hline & & Cornea & Viscous tear film & $50 \cdot 0$ \\
\hline & & & Mucous threads in tear film & $25 \cdot 0$ \\
\hline & & & Film attached to cornea & Io.0 \\
\hline & & & $\begin{array}{l}\text { Large mucous threads attached to } \\
\text { cornea }\end{array}$ & $5 \cdot 0$ \\
\hline & \multirow[t]{9}{*}{ After } & Conjunctiva & Triangular interpalpebral staining & $100 \cdot 0$ \\
\hline & & & CMarked & $15 \cdot 0$ \\
\hline & & & Intermediate & $65 \cdot 0$ \\
\hline & & & Scattered & $20 \cdot 0$ \\
\hline & & & Mucus attached to conjunctiva & $20 \cdot 0$ \\
\hline & & Cornea & $\begin{array}{l}\text { Punctate staining-including } \\
\text { staining of pits }\end{array}$ & $40 \cdot 0$ \\
\hline & & & Mucous threads in tear film & $25 \cdot 0$ \\
\hline & & & Filaments attached to cornea & $20 \cdot 0$ \\
\hline & & & $\begin{array}{l}\text { Large mucous threads attached to } \\
\text { cornea }\end{array}$ & \\
\hline \multirow{2}{*}{\multicolumn{2}{|c|}{ Fluorescein }} & Cornes & & \\
\hline & & Ciornea & & $30 \cdot 0$ \\
\hline
\end{tabular}




\section{(a) Replacement therapy}

On the basis that patients suffering from KCS tolerated alkaline solutions, Jones and Coop (1965) developed a tear substitute solution, B.J.6, with a $\mathrm{pH}$ of $8 \cdot 45$.

The $9^{8}$ patients in this study were issued with tear substitute drops for use no less than four times a day, the supply being renewed every 4 weeks. They were also instructed to bathe their eyes with decinormal saline night and morning to remove excess mucus.

\section{RESULTS}

64 patients (62 per cent.) completed 5 years of this therapy without receiving any other treatment; 44 of the 98 had fewer symptoms and signs than at the beginning of the trial. Although more patients showed subjective than objective improvement, nearly all of those whose signs diminished also had symptomatic relief. As each year passed, almost the same percentage of remaining patients required alternative or additional treatment; for example nineteen of 98 ( 18 per cent.) in the first year and seven of 64 ( 13 per cent.) in the fifth year (Table III; Fig. I). The reasons for changing this basic therapy were increased symptoms in five patients, signs in nine, and both in 26 ; there was recurrent lid infection in fifteen, and corneal ulceration in seven.

Table III Response to tear substitute therapy

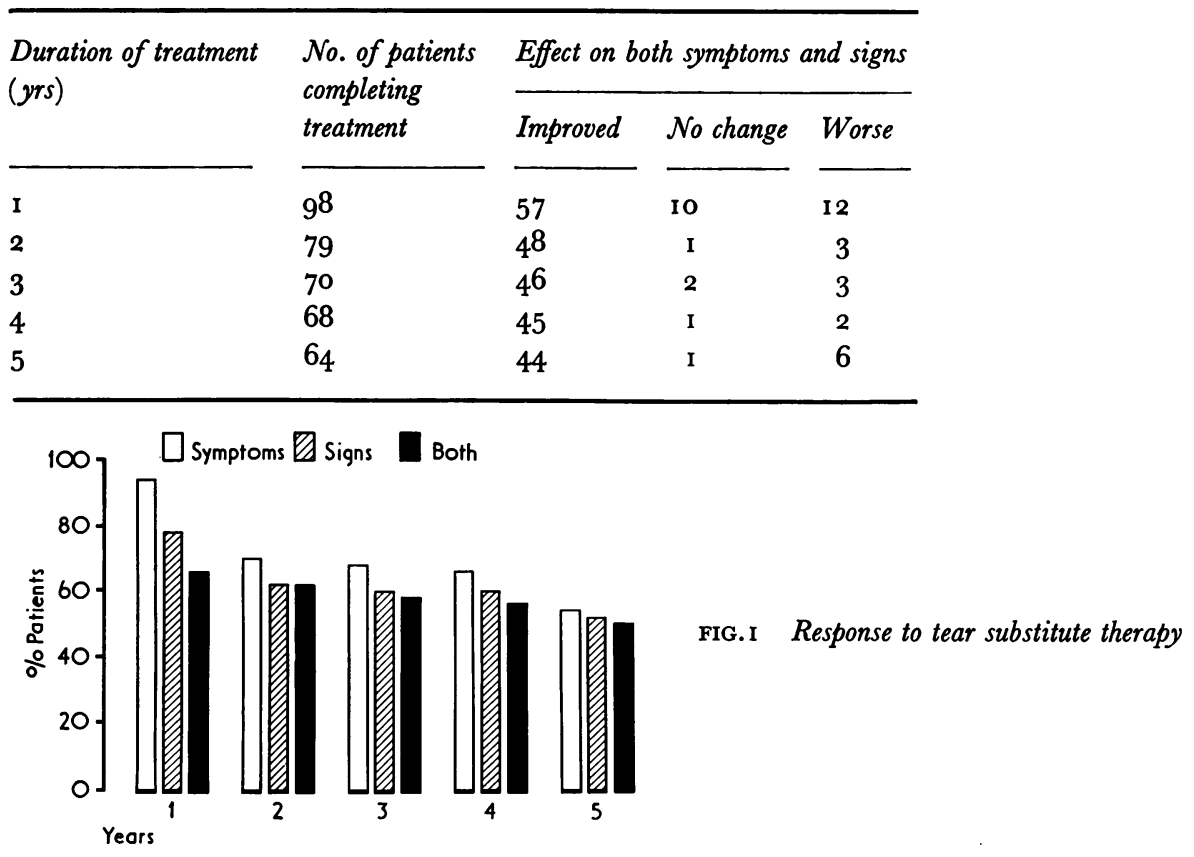

\section{(b) Conservation of tears (by nasolacrimal duct obliteration)}

Beetham (1935) was the first to destroy the nasolacrimal passages in patients suffering from KCS, so that the few tears being produced would be preserved for longer periods than usual. Mainly because of faulty selection of cases, his method, though excellent, fell into disuse. Holm (1949) suggested that symptoms and signs should have persisted for at least I year, and Jones and Coop (1965) emphasized that abnormal corneal epithelium should be present before this procedure was undertaken. 
Thirty ( 32 per cent.) of the 98 patients who had been on replacement therapy for at least I year were selected for nasolacrimal duct obliteration; 27 because of increased symptoms and signs and seven because of increased signs. In three patients the signs affected the conjunctivae only.

The tear passages, upper and lower, were obliterated by a diathermy probe using a current of $50 \mathrm{mAmps}$. When the overlying skin blanched the probe was removed and the punctum sealed. Generally 5 to Io sec. were sufficient for each canaliculus.

\section{RESULTS}

All thirty patients were followed for 3 years, at the end of which 25 ( 76 per cent.) were found to have benefited from this procedure, 2 I ( 57 per cent.) of them both subjectively and objectively. However, two patients continued to deteriorate and five were unaffected by the procedure. Ten of the thirty still required tear substitute therapy but were easier to control (Table IV; Fig. 2).

\section{Table IV Response to nasolacrimal passage obliteration}

\begin{tabular}{|c|c|c|c|c|}
\hline \multirow{2}{*}{$\begin{array}{l}\text { Duration of treatment } \\
\text { (yrs) }\end{array}$} & \multirow{2}{*}{$\begin{array}{l}\text { No. of patients } \\
\text { completing } \\
\text { treatment }\end{array}$} & \multicolumn{3}{|c|}{ Effect on both symptoms and signs } \\
\hline & & Better & No change & Worse \\
\hline I & 30 & 26 & 4 & I \\
\hline 2 & 30 & 22 & 6 & I \\
\hline 3 & 30 & $2 \mathrm{I}$ & 5 & 2 \\
\hline
\end{tabular}

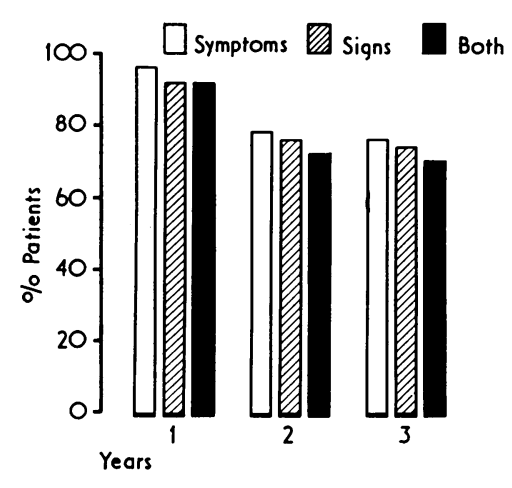

FIG.2 Response to nasolacrimal passage obliteration

\section{(c) Mucolytic agents}

Since excessive mucus may be partly responsible for the corneal changes in KCS and since corneal filaments appear to consist largely of mucin, one aim in the treatment of this condition should be the reduction of excess mucus (Jones and Coop, 1965).

$\mathrm{N}$-acetyl-cysteine has a reducing action of the free sulphydryl group of the mucoprotein molecules. This action finds widespread application in respiratory tract disease (Webb, I962), and in the short-term has been reported favourably in the treatment of KCS (Jones and Coop, r965; Absolon and Brown, r968; Messner and Leibowitz, 197 r).

Acetyl-cysteine has to be dispensed in plastic-topped bottles and may be diluted to a 5 per cent. solution with sodium bicarbonate added to a $\mathrm{pH}$ of 8.4 (the 20 per cent. solution 
appears to cause irritation, Absolon and Brown, 1968). The patients were instructed to instil the drops at least four times a day, to keep the bottles in the refrigerator, and to renew them after $48 \mathrm{hrs}$.

Twenty patients from our original group of 98 , displaying mucous shreds and corneal filaments after more than I year of replacement therapy, were selected for treatment with 5 per cent. acetyl-cysteine. Seven of these patients had had nasolacrimal duct occlusion without benefit. All were followed for I year.

\section{RESULTS}

There was no difference in subjective and objective improvement throughout the year. One patient was withdrawn after 2 months of therapy because her symptoms and signs were worse. At the close of the year eight patients (40 per cent.) had fewer symptoms, seven ( 35 per cent.) had fewer signs, and six (30 per cent.) had improved subjectively and objectively (Table V; Fig. 3).

Table V Response to acetyl-cysteine therapy

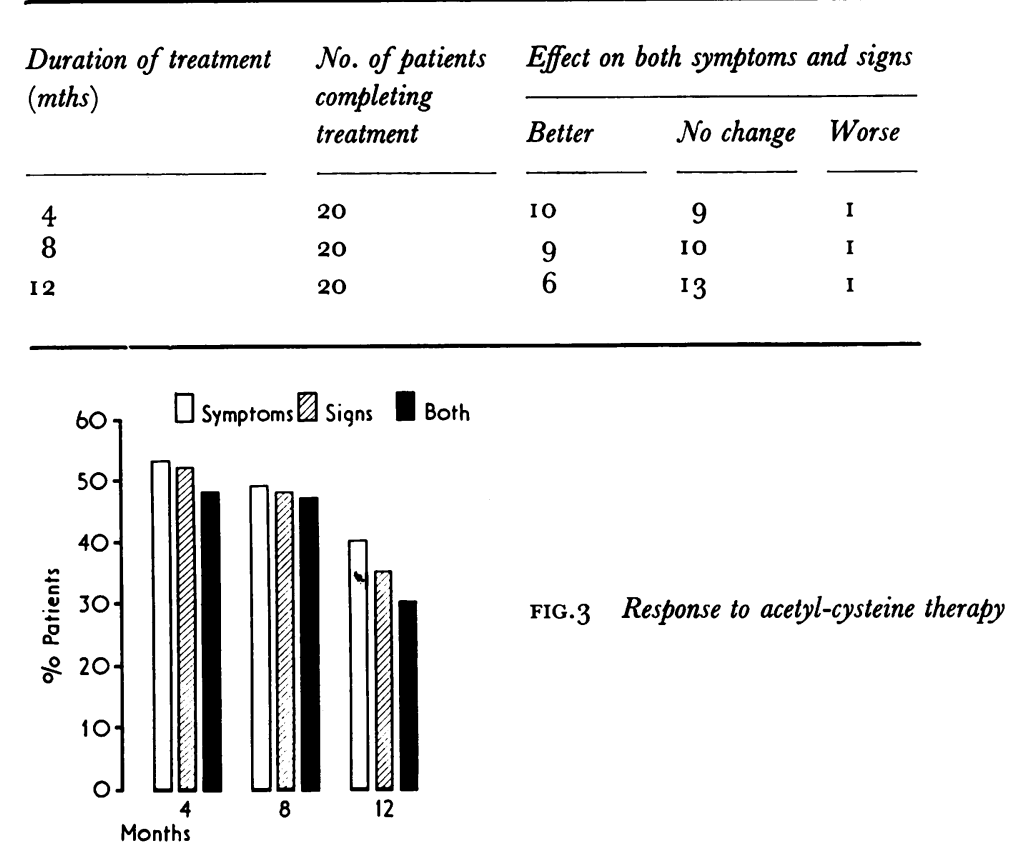

\section{(d) Hygiene, antibiotics, and topical steroids}

The dry eye is more susceptible to bacterial and fungal colonization than the healthy eye (Williamson, Gordon, Wood, Dyer, and Yaha, I968; Williamson, Wilson, Wallace, and Whaley, I97I $\mathrm{b}$ ), and patients must therefore practise strict personal hygiene. In this study the patients were instructed to use separate face-cloths and towels; the use of eyebaths was prohibited, and the dangers of contagion were emphasized.

Part of the regime of replacement therapy is twice daily bathing with dilute salt water prepared by the patient. When home conditions are inadequate, or the patient's personal hygiene is in doubt, it is better to forego this simple but effective measure. 
All of the newly-diagnosed patients, and those referred by ophthalmic colleagues, were treated with chloramphemicol drops four times a day and ointment at night for the first month of their supervision. Repeat cultures were performed on the patients suffering from blepharitis (Williamson and others, $197 \mathrm{Ib}$ ), and the antibiotic was continued until no bacteria could be detected. Nevertheless, 2 I of the $9^{8}$ patients in this series (almost 2 I per cent.) developed evidence of infection in the course of treatment with tear substitute therapy. Fourteen patients suffered from blepharitis and seven from various corneal lesions, and sixteen had recurrent bouts of infection requiring several courses of antibiotics.

Topical steroids were never prescribed for any of the patients in this trial, since we considered that any benefits were outweighed by the risks, a view held by other workers with considerable experience in this field (Wright, 1971).

\section{Discussion}

Before pronouncing on the efficacy of any treatment in cases of KCS, trials should be conducted over a prolonged period.

In this study, which took place between 1965 and 1972,98 patients suffering from Sjøgren's syndrome, were supervised for no less than 5 years. Although the calculation of subjective improvement presents difficulties because so many factors are involved, such as the patient's personality, home commitments, weariness after a long ambulance journey, etc., some record of the patient's symptoms is helpful, even if it indicates only a general trend. We recorded change in symptoms, based on a table of symptomatology developed for use in the KCS index (Williamson and others, 1971a). Furthermore, we based our objective findings on a table of biomicroscopic changes prevalent in patients suffering from Sjøgren's syndrome.

More patients responded subjectively than objectively to all the forms of treatment utilized in this series. It is significant, however, that nearly all those whose signs diminished also had symptomatic relief. Tear substitute therapy improved both symptoms and signs for 5 years in nearly $5^{0}$ per cent. of the $9^{8}$ patients. Each year about 16 per cent. of the remaining patients required alternative or additional treatment, indicating that tear substitute therapy alone would be unlikely to control the disease indefinitely (Fig. I).

In the second phase of this study, thirty patients were selected for nasolacrimal duct obliteration because they had not responded to simple tear substitute therapy. Of these, 25 derived benefit, 2 I (57 per cent.) both subjectively and objectively. After a period of 3 years, $4^{8}$ per cent. remained the better for this procedure, 70 per cent. both subjectively and objectively (Table IV; Fig. 2).

Holm (1949), who performed this operation in 23 cases and observed the long-term results in fourteen patients, found that eight ( 57 per cent.) were still much better after 5 to 6 years. Although Holm was impressed by the relief of symptoms he detected little difference in signs. In the majority of his cases, filaments, mucous shreds, and extensive staining with rose Bengal persisted. Only six patients ( 18 per cent.) in the present series improved symptomatically without changes in signs. Furthermore, Jones and Coop ( 1965 ) thought that, provided abnormal corneal epithelium was present, occlusion of lacrimal outflow was followed by improvement in both symptoms and signs. One of their failures had only slight corneal staining and had more trouble with epiphora after the operation than with the symptoms of dry eyes beforehand. In the present study, three patients with extensive conjunctival staining but no corneal activity were successfully treated and have been followed for 3 years. There would appear to be three possible explanations for this apparent contradiction of Jones and Coop's criteria : 
(1) Abnormal corneal epithelium may have been present between visits to the clinic.

(2) The KCS was saved from progressing to corneal involvement only by occlusion of the nasolacrimal ducts.

(3) Abnormal corneal epithelium may not be the decisive factor influencing the choice of this operation.

It may be noted that mucous shreds and extensive conjunctival staining may be present in the absence of corneal filaments but in the presence of marked symptoms. In addition, Holm (1949) noted that, even in patients who showed considerable symptomatic relief after occlusion of nasolacrimal overflow, corneal filaments might persist. In our study, six patients gained symptomatic relief with no change in signs. This phenomenon supports the view that visibly abnormal corneal epithelium need not be the only criterion for the operation and that resistance of symptoms should be regarded as of prime importance.

Ten of the successfully treated patients required the continued use of tear substitute therapy, making it apparent that more than one method of treatment may be necessary in selected cases.

Twenty patients from our original group of 98 were chosen for acetyl-cysteine therapy because they had failed to respond to tear substitute therapy and/or nasolacrimal duct obliteration. In this context it is interesting to observe that Absolon and Brown (1968) thought that some of their patients might not have been able to distinguish discomfort caused by the acetyl-cysteine from symptoms due to the disease. Furthermore, British Drug Houses Ltd., who manufacture acetyl-cysteine, recommended dilution to 1o or 5 per cent. to reduce ocular irritation. Finally, the $\mathrm{pH}$ of the solution in our study was adjusted to $8 \cdot 4$ since mucus is dissolved more readily in alkaline solution. Over a period of I year, six patients ( 30 per cent.) showed both objective and subjective improvement, i.e. 6 per cent. of the original group of $9^{8}$ patients. Jones and Coop ( 1965 ) reported striking relief of symptoms in thirteen of fifteen patients (87 per cent.), but the duration of their observations is not stated. Absolon and Brown reported more improvement in the signs in thirty patients treated for 2 months with acetyl-cysteine than with B.J.6, but no difference in symptoms was reported.

It is clear that this highly selective body of patients represents a resistant form of KCS. Since acetyl-cysteine is a mucolytic agent, only patients who displayed corneal filaments and/or mucous threads were chosen for treatment. Therefore this group of patients may have been examples of a more severe degree of KCS than those reported by Jones and Coop ( 1965 ) and Absolon and Brown (1968). All the patients in the present group had definite Sjøgren's syndrome. The associated diseases in Jones and Coop's group were not annotated, and only 22 of the thirty patients in Absolon and Brown's study had rheumatoid arthritis. In addition, these investigators prescribed 20 per cent. acetyl-cysteine at a $\mathrm{pH}$ of 7 .

Nevertheless, the results of the present investigation are gratifying in that 30 per cent. of twenty patients who were untouched by previous treatment reacted favourably to 5 per cent. acetyl-cysteine.

Both Jones and Coop (1965) and Wright (1971) observed that recurrent infection, particularly with Staphylococcus aureus, was so common in KCS as to require constant vigilance. In a previous study (Williamson and others $197 \mathrm{I}$ b), the prevalence of bacterial isolates, pathogenic staphylococci in particular, was shown to be higher on the lid margins and in the conjunctival sacs of untreated KCS patients, than in a control series of rheumatoid arthritic patients. Overt blepharitis, sometimes accompanied by folliculitis, styes, or yellow conjunctival discharge, was present in about 30 per cent. of newly diagnosed cases, and the prevalence of Staphylococcus aureus was highest of all in this group. Most of the staphylococci were resistant to penicillin but not to chloromycetin or tetracycline. 
For these reasons all of the newly diagnosed patients and those referred by ophthalmic colleagues were treated with chloramphenicol drops four times a day and ointment at night for the first month of their supervision. Repeat cultures were performed in these patients suffering from blepharitis until no bacteria could be detected. Nevertheless 2 I of the 98 patients (nearly 21 per cent.) developed evidence of infection during treatment with tear substitute therapy.

The tendency to recurrent infection in a small number of cases indicates the need for constant vigilance. Although severe corneal complications are said to be uncommon in Sjøgren's syndrome, a large series, such as the present one, is bound to include a fair number, particularly after a long period. The relation between these complications and bacterial, fungal, and viral infections will be the subject of a future communication.

\section{Summary}

98 patients suffering from Sjøgren's syndrome were supervised for not less than 5 years, their ocular progress being carefully documented. Both symptoms and signs improved in nearly 50 per cent. of the group receiving tear substitute therapy only for the period of 5 years. Thirty of the 98 patients were selected for nasolacrimal canalicular obliteration, and 70 per cent. of these continued to improve both objectively and subjectively over the follow-up period of 3 years. Twenty of the 98 patients who had not responded to tear substitute therapy or nasolacrimal canalicular obliteration were treated for I year with 5 per cent. acetyl-cysteine in solution $\mathrm{pH} 8 \cdot 4.30$ per cent. of this group improved in both symptoms and signs. Recurrent infection, particularly with Staphylococcus aureus on the lids, is a problem requiring constant vigilance and may be related to the development of corneal complications.

\section{References}

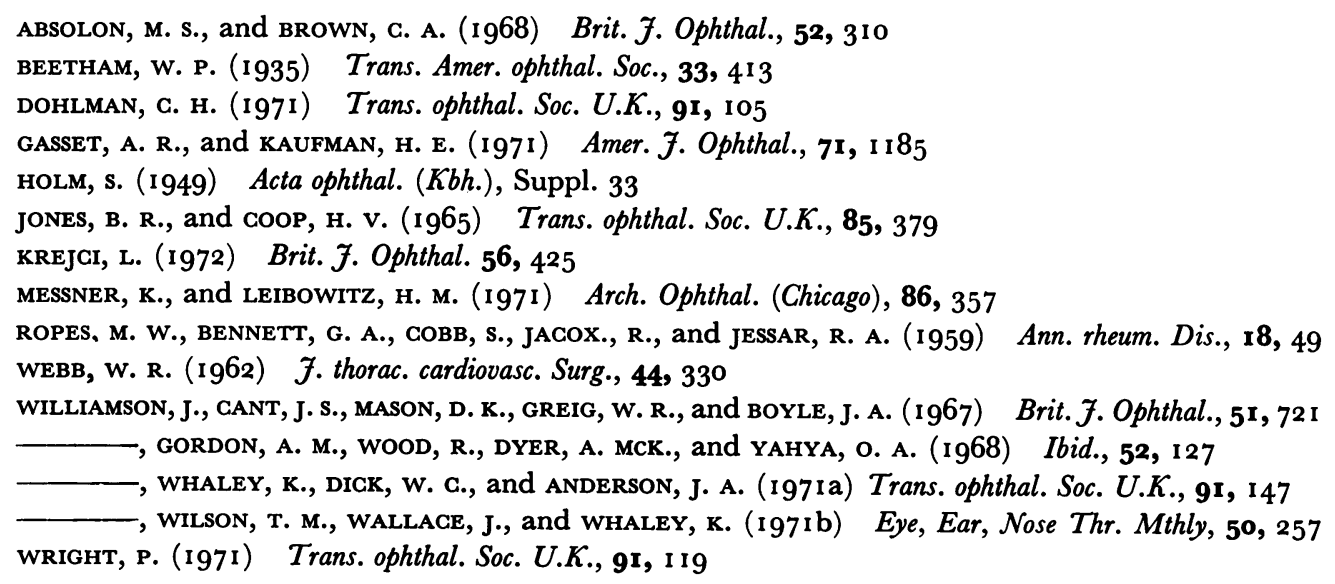

